\title{
Die Autonomie des psychosomatischen Patienten
}

\author{
Hilfesuchender Patient oder autonomer Kunde? Dieses Thema der letzten SÄZ-Podi- \\ umsdiskussion birgt bei psychisch und psychosomatisch kranken Patienten beson- \\ dere Probleme. Die Klärung verschiedener Missverständnisse ist notwendig, damit \\ auch für diese Patienten sinnvolle Rahmenbedingungen in unserem Sozialversiche- \\ rungssystem erhalten werden können.
}

\section{Marianne Schneider Weber}

Fachärztin für Psychiatrie und Psychotherapie FMH, Psychosomatische Medizin SAPPM, Dozentin IHM, Lehrbeauftragte Universität Zürich
Im Text wird die männliche und weibliche Form abwechselnd verwendet.

Korrespondenz:

Dr. med.

Marianne Schneider Weber Kronenstrasse 42

CH-8006 Zürich

marianne@schneiderweber.ch

www.ihm-institut.ch

\section{Einleitung}

In Ergänzung zu dem anregenden Artikel von Professor Gerhard Rogler «Der Patient als Kunde» [1] und von Felicitas Witte über die Basler Podiumsdiskussion der SÄZ zum Thema «Kunden oder Patienten - Medizin zwischen Dienstleistung und Fürsorge» [2] sollen hier spezifische Auswirkungen des aktuellen politischökonomischen Umfeldes auf die Arzt-Patienten-Beziehung von psychosomatisch und psychisch kranken Patienten diskutiert werden. Während Gerhard Rogler den Mangel an Fürsorge vor allem für die chronisch oder schwerkranken Patienten beklagt, also für eine Patienten-Gruppe, die offensichtlich als bedürftig erscheint, ist es sehr viel schwieriger, die Konsequenzen der Ökonomisierung der Arzt-Patienten-Beziehung für eine Patientengruppe nachvollziehbar darzustellen, deren Leiden weniger sichtbar und gesellschaftlich tabuisierter ist. Diese Patienten fallen zwar auch oft durch die Maschen des marktwirtschaftlich gewinnorientierten Medizinalsystems, die Lage der Psychosomatiker ist jedoch komplexer. Denn immer häufiger tritt auch der psychosomatisch oder psychisch Kranke nicht als Hilfesuchender, Bedürftiger auf, sondern als pseudoautonomer Kunde, der seine Ansprüche geltend macht. Er drängt seinen Dienstleister/Arzt zu Untersuchungen, Therapien usw., die ihm mehr schaden als nützen. Das führt zu einer weiteren, sinnlosen Ressourcenvergeudung.

\section{Autonomie und ihre Einschränkungen}

Versteht man Autonomie als Willensfreiheit, bzw. als «angeeigneten Willen» [3], beginnt sie zunächst mit der Artikulation dessen, was man will, vor allem langfristig will. Nicht alle Menschen entwickeln einen freien Zugang zu ihren eigenen Wünschen, können diese realistisch bewerten und in ihrem Leben als personale Autonomie integrieren. Auch der Gesunde wird immer wieder um diese personale Autonomie ringen müssen. Autonomie ist kein unerschütterlicher, fester Bestandteil unserer Psyche, der, einmal erworben, nicht zeitweilig wieder eingeschränkt werden könnte. Jeder Arzt hat an sich selbst schon die Erfahrung ge- macht, dass Ängste, wie z. B. ein unklarer Befund, die Entscheidungsfreiheit beeinträchtigen und ein Gefühl von Abhängigkeit aufkommen lassen.

Ein konkreter Fall: Eine psychosomatisch kranke Patientin klagt im Rahmen der hausärztlichen Betreuung über rezidivierende Unterbauchbeschwerden und drängt auf eine umfassende Abklärung. Was bedeutet es hier, die Patientenautonomie zu respektieren bzw. den Patienten als autonomen Kunden zu betrachten?

Oder wie sieht es aus mit der autonomen Entscheidungsfähigkeit beim Abschluss eines Therapievertrags für eine psychiatrisch-psychotherapeutische Behandlung eines Borderlinepatienten, der «einfach seine Sucht weghaben» möchte?

Gemäss dem Konzept des Informed Consent, der unsere klassische hippokratische Fürsorgeethik erweitert, ist die Voraussetzung für eine Behandlung die $\mathrm{Zu}$ stimmung (consent) als autonomer Akt eines wohlinformierten Patienten, der damit eine Art Konsumentenvertrag mit dem Dienstleister-Arzt eingeht [4].

Nun kann aber die erforderliche Patientenautonomie, die persönliche, emotionale und geistige Autonomie respktive die Willensfreiheit für eine solche Entscheidung in der Therapie auf vielfältige Weise eingeschränkt sein, z. B. durch unrealistische Vorstellungen und Erwartungen an Behandlungen oder durch eine unangemessene Selbsteinschätzung. Nicht nur deutlich depressive oder ängstliche Patienten neigen $\mathrm{zu}$ Fehleinschätzungen ihrer Symptomatik, vor allem persönlichkeitsgestörte Patienten (ca. 10\% der Bevölkerung) mit Ich-strukturellen Problemen [5] sind oft nicht imstande, das Ausmass ihrer Störung wahrzunehmen, und verleugnen ihre Verhaltensauffälligkeiten (oft selbstdestruktiver Art), für die sie auch keine Verantwortung übernehmen (können). Diese Beeinträchtigungen ihrer emotionalen Funktionsfähigkeit $[6,7]$ sind häufig die Folge früher Bindungsstörungen oder Traumatisierungen. $\mathrm{Zu}$ ihnen zählen nicht nur psychiatrische, sondern auch viele Patienten mit Somatisierungs- und somatoformen Störungen, deren Problematik oft ungenügend wahrgenommen wird. 


\section{«Mangelnde Information» - ein Missverständnis}

Medizinische Laien, wie viele Politiker und Ökonomen, mögen sich nun vorstellen, dass diese Patienten einfach besser informiert werden müssen, und verwechseln solche Einschränkungen mit intellektuellen, bildungsbedingten Defiziten. Erika Ziltener vom Dachverband Schweizerischer Patientenstellen spricht am erwähnten SÄZ-Symposium z.B. von Leuten, die sich im medizinischen System nicht gut auskennen, etwa ter, als ein auf Kunden ausgerichteter Verkäufer von medizinischen Angeboten, so wird er einen solchen Patienten bedienen oder wird seinen Zweifel am Nutzen seiner Dienstleistung besänftigen und sich auf den autonomen, aufgeklärten Patienten berufen. Bestimmt generieren gerade diese fordernd auftretenden, oft mit Internetinformationen «bewaffneten» pseudoautonomen Patienten mit unsinnigen Untersuchungen mehr Kosten als klärende Gespräche von psychosomatisch ausgebildeten Grundversorgern.

\section{Es besteht nicht nur die Gefahr, dass fordernde Patienten durch sinnlose Untersuchungen unser Sozialversicherungssystem aushöhlen, sondern auch, dass die Behandler selbst korrumpiert werden}

Schreiner oder Verkäuferinnen. Doch es gibt viele einfache, wenig gebildete Patienten, die in gutem emotionalem Kontakt mit sich stehen und durchaus imstande sind, auch in komplizierten medizinischen Situationen sinnvoll für sich zu entscheiden.

Ganz anders bei Patienten mit psychischen oder psychosomatischen Leiden, deren Problematik primär auf emotionaler Ebene liegt und die auch durch geduldige medizinische Information (Vor- und Nachteile von medizinischen Interventionen) nicht einfach «autonom gemacht» werden können. Sie lassen sich nicht durch logische Argumente oder durch statistische bzw. probabilistische Aussagen überzeugen. So neigen auch durchaus engagierte Hausärzte gelegentlich dazu, viel auf solche Patienten einzureden, ohne zu merken, dass dies nichts nützt, sondern im Gegenteil die emotionale Verwirrung steigert und die Aufnahmebereitschaft solcher Patienten senkt.

\section{Die Pseudoautonomie}

Gewisse psychosomatisch Kranke, vor allem ausgeprägt narzisstische, leiden unter ihrer Abhängigkeit. Ihre eingeschränkten Möglichkeiten, eigene Gefühle differenziert wahrzunehmen, führen dazu, dass sie oft sehr aussen- oder fremdgeleitet handeln. Sie tendieren dazu, sich in nahen Beziehungen übermässig anzupassen oder sich gar zu unterwerfen. Dadurch werden ihre Beziehungen belastend und ihr Leben orientiert sich am Konkreten. In Auflehnung gegen diese Beeinträchtigung entwickeln viele eine Art kompensatorischer Pseudoautonomie.

Sie verhalten sich dann besonders uneinsichtig und abweisend gegen Hilfe und machen es ihren Behandlern schwer, eine Art Hilfsfunktion zu übernehmen. Nicht selten versuchen sie, Mitmenschen für ihre Zwecke zu manipulieren, fordern z. B. Rezepte, Zeugnisse usw. oder erheben Anspruch auf Behandlungen nach eigenen Vorstellungen.

Wird dieses Verhalten als echte Autonomie missverstanden, oder versteht sich der Arzt als Dienstleis-
Die Behandlung solcher Patienten braucht kommunikatives Geschick und fachliche Kompetenz. Wenn es gelingt, ihr Vertrauen zu gewinnen, können sie ihren Arzt als eine Art ihnen zur Verfügung stehendes «Hilfs-Ich» akzeptieren. Oft können sie erst mit Hilfe eines solchen ärztlichen «Containings» [8] ihre emotionalen Einschränkungen wahrnehmen.

Das Vertrauen in ihre Mitmenschen ist bei Patienten mit ausgeprägten ich-strukturellen Problemen nicht nur wegen ihrer Intoleranz gegenüber Abhängigkeit gefährdet, sondern auch deshalb, weil sie die Intentionen anderer Menschen schlecht einschätzen können. Das ist mit ein Grund, weshalb es häufig zu Beziehungsabbrüchen kommt, was auch die Arzt-Patienten-Beziehung gefährdet und sich häufig erst mit einer psychotherapeutischen Behandlung verbessern lässt.

\section{Die eingeengte Arzt-Patienten-Beziehung}

Viele Ärzte fühlen sich durch Kontrolle, Bürokratisierung, Evidence based medicine zunehmend unfreier und ziehen sich auf die Rolle als Dienstleister zurück. Sie fokussieren auf die zu behandelnde Störung und nehmen den Patienten nicht mehr als Subjekt mit eigenem Erleben wahr. Die subjektive Leidensgeschichte, die emotionale Botschaft bei der Beschreibung der somatischen Symptomatik kann vom Dienstleister nicht aufgenommen, reflektiert und verstanden werden.

Die Einengungen des Arztes kumulieren mit den Einengungen durch die emotionale Problematik des Patienten. Die Ärztin kann die so wichtige Hilfs-IchFunktion nicht mehr übernehmen. Die Verstrickung zwischen Arzt und Patientin steigt, er wird affiziert von den Ängsten und Druckgefühlen dieser Patienten und erliegt ebenfalls dem konkretistischen Kurzschluss, nämlich Symptom X erfordere Handlung Y. Ein Circulus vitiosus von unnötigen Aktionen und zunehmender Resignation setzt ein, er wird instrumentalisiert, oder es kommt zu Weiterweisungen. 
Im Kunden-Dienstleister-Denken sind solche Schwierigkeiten kein Thema, die therapeutischen Misserfolge werden nicht mehr im Kontext der Arzt-Patienten-Beziehung betrachtet, als mangelnde Adherence (früher Compliance genannt) oder als intersubjektive «Inszenierungen» von Patient und Arzt. Man beschränkt sich auf die Frage, wie man solche «schlechten Risikopatienten» besser managen oder von Sozialleistungen ausschliessen kann.

Wenn nun die IV keine Beiträge an Patienten mit somatoformen Störungen mehr bezahlt, dann ist wohl endgültig klar, wer die Hauptverlierer unseres durchökonomisierten Gesundheitssystems zu werden drohen.

\section{Spielräume und hilfreiche Dritte}

Wie kann der Verengung entgegengetreten werden? Wie kann ein bio-psycho-sozialer Zugang zu diesen Patienten geschaffen werden?

Im Gespräch mit einem Dritten, mit der Arztgehilfin, einem anderen Behandler, in einer Balint- oder Supervisionsgruppe kann oft der notwendige Abstand zum Erlebten wieder geschaffen werden, und neue Perspektiven eröffnen sich.

Doch diese Erkenntnisse brauchen Zeit, Raum und wohl auch etwas Freude und Interesse an der menschlichen Verrücktheit! Auch Kreativität, Humor und unkonventionelles Denken sind Eigenschaften, welche die Chancen auf einen Zugang zu solchen Patienten verbessern. Am wichtigsten ist wohl die innere Ruhe, Ängste und Ohnmachtsgefühle der Patienten zu ertragen, bis diese selbst zunehmend mehr Verantwortung übernehmen und sinnvolle Behandlungen akzeptieren können.

Doch welche Qualitätssicherungsfragebogen erfassen solche ärztlichen Eigenschaften? Wie weit lassen sich obenerwähnte Eigenschaften mit den ständig steigenden Leistungsansprüchen und geforderten Managerqualitäten an Ärztinnen verbinden?

Patienten mit psychosomatischen Leiden treten oft sehr fordernd auf und lassen sich durch rein logische Argumente schwer von gewünschten, aber unnötigen Behandlungen abbringen.

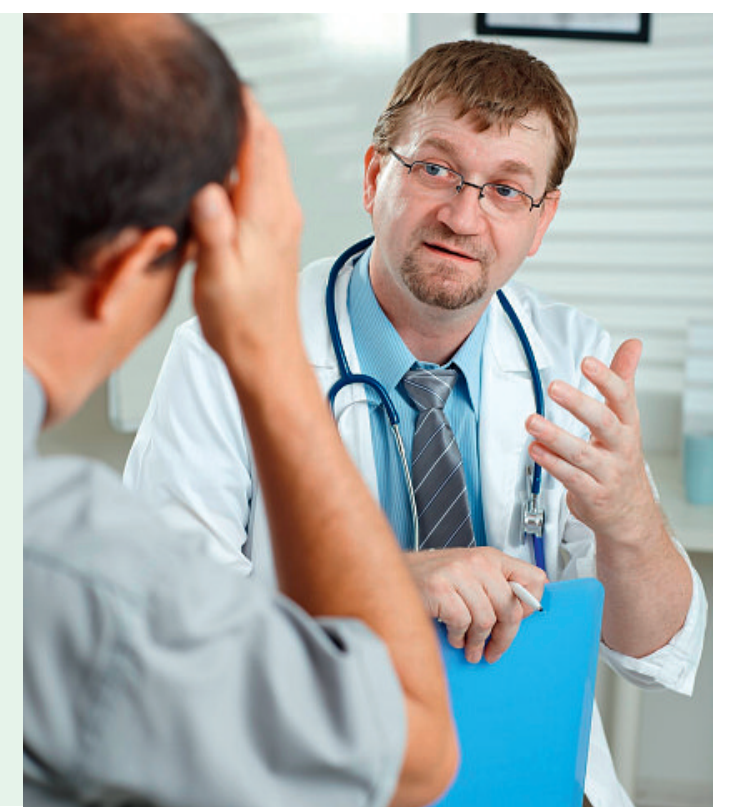

\section{Dranbleiben oder Kapitulieren?}

Durch die Ökonomisierung der Medizin verändert sich die Arzt-Patienten-Beziehung schleichend. Es besteht nicht nur die Gefahr, dass anspruchsvolle und fordernde Patienten durch sinnlose Untersuchungen unser Sozialversicherungssystem aushöhlen, sondern auch, dass die Behandler selber korrumpiert werden.

Zwar sind die Möglichkeiten der Behandlung dieser Patienten vielfältig und nicht erfolglos, aber die Möglichkeiten des Scheiterns sind auch beachtlich. So sind die Ohnmachtsgefühle schwer erträglich, die Konsultationen unregelmässig, und das Honorar für «verpasste Stunden» gelegentlich schwer einzutreiben, d.h. diese Patienten stellen auch einen ökonomischen Risikofaktor dar.

Sind Psychosomatiker also nichts für einen kalkulierenden Leistungserbringer? Oder werden bald nur noch tariflich versierte Kolleginnen und Kollegen Wege finden, Kapital aus psychosomatischen Patienten zu schlagen, ohne sich über die Sinnlosigkeit ihrer Aktivitäten den Kopf zerbrechen zu müssen oder von santésuisse zurückgepfiffen zu werden? Wird das Credo der Autonomie des aufgeklärten Patienten den Dienstleister von Selbstzweifeln, Fürsorgeaufgaben und «unangenehmen Gefühlen», die über tarifierte Zeit hinausgehen, befreien?

Oder gelingt es doch, mit Engagement, Argumentation und ständiger Aufklärung der von den Patientenrealitäten entfernten Ökonomen sinnvolle Rahmenbedingungen in unseren Sozialversicherungssystemen aufrechtzuerhalten, die eine differenzierte und hilfreiche Gestaltung der Arzt-Patienten-Beziehung ermöglichen?

\section{Literatur}

1 Rogler G. Der Patient als Kunde? Schweiz Ärztezeitung. 2009;90(25):1009-13.

2 Witte F. Kunden oder Patienten - Medizin zwischen Dienstleistung und Fürsorge. Schweiz Ärztezeitung. 2009;90(51/52): 2001-3.

3 Bieri P. Das Handwerk der Freiheit. München, Wien: Carl Hanser; 2001.

4 Tress W, Erny N. Patientenautonomie ein dynamisches Konzept. PiD-Psychotherapie im Dialog. 2009;10:291-95.

5 Ich-strukturelle Störungen manifestieren sich als Ich-Schwäche, Identitätsdiffusion, eingeschränkte Fähigkeit zur Realitätsprüfung und Tendenz $\mathrm{zu}$ instabilen Beziehungen.

6 Fonagy P. Gergely G, Jurist EL, Target M. Affektregulierung, Mentalisierung und die Entwicklung des Selbst. Stuttgart: Klett-Cotta; 2004.

7 Schlecht entwickelte emotionale «Theory of Mind»-Funktionen oder Mentalisierungsfähigkeiten führen dazu, dass eigene und fremde Emotionen falsch wahrgenommen, reflektiert und interpretiert werden können.

8 Eine haltende, akzeptierende Beziehung, die dem Patienten ermöglicht die eigenen Gefühle zu klären. 